\title{
Green-pumped, picosecond MgO:PPLN optical parametric oscillator
}

\author{
Florian Kienle, ${ }^{1, *}$ Dejiao Lin, ${ }^{1}$ Shaif-ul Alam, ${ }^{1}$ Hazel S. S. Hung, ${ }^{2}$ Corin B. E. Gawith, ${ }^{2}$ \\ Huw E. Major, ${ }^{2}$ David J. Richardson ${ }^{1}$, David P. Shepherd ${ }^{1}$ \\ ${ }^{1}$ Optoelectronics Research Centre, University of Southampton, Highfield, Southampton, SO17 \\ $1 B J, U K$ \\ ${ }^{2}$ Covesion Ltd., Romsey, SO51 9DG, UK \\ *Corresponding author: flk@orc.soton.ac.uk
}

\begin{abstract}
We investigate the performance of a magnesium-oxide-doped periodically poled lithium niobate crystal (MgO:PPLN) in an optical parametric oscillator (OPO) synchronouslypumped by $530 \mathrm{~nm}, 20 \mathrm{ps}, 230 \mathrm{MHz}$ pulses with an average power of up to $2 \mathrm{~W}$ from a frequency-doubled, gain-switched laser diode seed and a multi-stage Yb:fiber amplifier system. The OPO produces $\sim 165 \mathrm{~mW}$ (signal, $845 \mathrm{~nm}$ ) and $\sim 107 \mathrm{~mW}$ (idler, $1421 \mathrm{~nm}$ ) of average power for $\sim 1 \mathrm{~W}$ of pump power and can be tuned from $\sim 800 \mathrm{~nm}$ to $900 \mathrm{~nm}$ (signal) and $1.28 \mu \mathrm{m}$ to $1.54 \mu \mathrm{m}$ (idler). Observations of photo-refraction and green-induced infrared absorption (GRIIRA) in different operational regimes of the MgO:PPLN OPO are described and the role of peak intensity and average power are investigated, both with the aim to find the optimal operating regime for pulsed systems.
\end{abstract}

OCIS codes: 190.0190, 190.4970, 190.4400, 140.7090, 060.2320.

\section{Introduction}

Tunable ultrashort pulses in the visible (VIS) and near-infrared (NIR) spectral range have a multitude of applications including coherent anti-Stokes Raman scattering (CARS) spectroscopy [1-4] and stimulated-emission-depletion (STED) microscopy [5]. Ti:Sapphire lasers have been the source-of-choice in the past, but cheaper, more compact, user-friendly alternatives covering this wavelength region are increasingly sought-after. In CARS, synchronously-pumped OPOs are an ideal choice as they emit two temporally synchronous pulses at different (and tunable) wavelengths. The pump sources used for these OPOs are typically ultrashort solid-state laser systems operating at $\sim 1 \mu \mathrm{m}$ and frequency-doubled to the green.

For green-pumped OPOs that produce NIR wavelengths, bulk crystals such as lithium triborate (LBO) offer high damage thresholds and wide transparency ranges, but only moderate values of effective nonlinearity (gain). LBO has long been the preferred nonlinear crystal for high-power, pulsed OPOs, mainly due to its high damage threshold of $>10 \mathrm{GW} / \mathrm{cm}^{2}$ and the possibility of non-critical birefringent phase-matching [6-9]. The highest signal output power recorded from a picosecond LBO OPO of $1.6 \mathrm{~W}$ tunable from $744 \mathrm{~nm}$ to $930 \mathrm{~nm}$ was reported by Tukker et al. [9] using 5.6W of pump power from a frequency-doubled, 50ps Nd:YLF laser. Master-oscillator power-amplifier (MOPA) configurations based on ytterbium-doped fibers (Yb:fiber), rather than bulk solid-state laser systems, have also been used as pump sources in recent years including reports of picosecond [4] and femtosecond [1] systems delivering hundreds of milliwatts of OPO average output power with signal tunability over $\sim 200 \mathrm{~nm}$ in the NIR. 
Quasi-phase-matched (QPM) materials such as periodically-poled lithium niobate (PPLN), lithium tantalate (PPLT) and potassium titanyl phosphate (PPKTP) offer large nonlinear coefficients and are a popular choice for $1 \mu \mathrm{m}$-pumped OPOs for mid-infrared (MIR) generation. However, in the presence of visible light, common QPM materials are more sensitive to optical damage mechanisms such as photo-refraction, GRIIRA, nonlinear absorption and/or greytracking, which at high pulse energies can result in reduced conversion efficiencies, degraded beam qualities, higher oscillation thresholds, temporal instabilities or even physical crystal damage. Photo-refractive effects cause a distorted refractive index profile [10-12]. High intensities in the VIS generate free charge carriers through photo-ionization and the associated electric field induces a refractive index distortion via the linear electro-optic effect. In the case of lithium niobate (LN), the induced absorption due to GRIIRA originates from color center creation, crystal impurities (mainly Fe-ions), intrinsic defects ( $\mathrm{Nb}$-ions occupying Li-ion sites) and the polaron effect [13-15]. Since the absorption of the NIR power grows quadratically with incident green power [13], GRIIRA can become a major problem when power-scaling OPOs. Furthermore, Hirohashi et al. report that both linear and nonlinear (e. g. two-photon) absorption, the latter especially in picosecond experiments, can occur in ferroelectric materials such as LN [15]. Impurities and defects can lead to linear absorption, whereas nonlinear absorption is possible because of the specific band gap of ferroelectrics. In any case, the absorbed energy leads to thermal lensing and consequently resonator instability and degraded beam quality.

With improved material properties and advanced fabrication techniques, photo-refraction and GRIIRA in LN can be reduced resulting in an increased damage threshold. To improve the performance of LN under the influence of high intensities in the VIS, a stoichiometric composition [12], where the concentration of $\mathrm{Li}$ - and $\mathrm{Nb}$-ions is balanced, can be used. Alternatively, $\mathrm{MgO}$-doping of congruent material, where $\mathrm{Mg}$-ions replace $\mathrm{Nb}$ anti-site defects [16], has similar performance benefits. MgO:PPLN has been successfully used in green-pumped CW OPOs $[17,18]$ as well as in CW second-harmonic generation (SHG) experiments with green average powers of up to $3 \mathrm{~W}$ for shorter periods of time [19] and $2.2 \mathrm{~W}$ over more than $2000 \mathrm{~h}$ [20]. However, due to the onset of photo-refraction and GRIIRA and the high peak powers, there has been limited research activity with ultrashort-pulsed, green-pumped OPOs based on LN. Some work with PPLN in the ultrashort-pulse regime has been carried out previously [21] resulting in the demonstration of $\sim 2 \mathrm{ps}, 120 \mathrm{MHz}$ pulses at a quasi-CW power of $140 \mathrm{~mW}$ with a total tuning range from $883 \mathrm{~nm}$ to $1285 \mathrm{~nm}$ using a mode-locked Nd:YLF laser and amplifier, whose output is frequency-doubled using LBO. However, to access higher peak powers in the kilowatt range from the amplifier, $10 \mu \mathrm{s} / 2 \mathrm{kHz}$ pulses were sliced from the seed picosecond pulse train and therefore the average power into the crystal was $50 \times$ reduced. More recently, MgO:PPLN has been used in a picosecond, $80 \mathrm{MHz}$, green-pumped OPO for CARS spectroscopy producing $\sim 30 \mathrm{~mW}$ of signal and $\sim 30 \mathrm{~mW}$ of idler power over a tunability range of $880 \mathrm{~mm}$ to $1040 \mathrm{~nm}$ and $1090 \mathrm{~nm}$ to $1350 \mathrm{~nm}$, respectively [22].

LN remains an interesting candidate for green-pumped, ultrashort-pulse OPOs, because of the above-mentioned material improvements and its well-known attractive QPM properties (high effective nonlinear coefficient $d_{\text {eff }}=17 \mathrm{pm} / \mathrm{V}$ and designer phase-matching [23]), wellunderstood physical and optical properties, wide availability and low price. Previously, we have shown that MgO:PPLN is well suited for ultrashort OPOs pumped at $1 \mu \mathrm{m}$ operating at high average powers $>10 \mathrm{~W}$ [24] and at high pulse energies $>0.5 \mu \mathrm{J}$ [25]. In addition to this, experiments performed at Covesion Ltd. have demonstrated CW SHG with MgO:PPLN generating $>2 \mathrm{~W}$ of green $\left(>200 \mathrm{~kW} / \mathrm{cm}^{2}\right)$ without any impairments in performance over $2000 \mathrm{~h}$ [20]. Consequently, we now want to investigate the optimal operation regime for MgO:PPLN when used in ultrashort-pulse, green-pumped OPOs. 
In this contribution, we demonstrate an MgO:PPLN OPO synchronously pumped with $530 \mathrm{~nm}, 20 \mathrm{ps}, 230 \mathrm{MHz}$ pulses from a frequency-doubled, fiber-amplified, gain-switched laser diode MOPA system producing $\sim 270 \mathrm{~mW}$ of combined output power for $\sim 1 \mathrm{~W}$ of pump power. We discuss the limiting effects of photo-refraction, GRIIRA and nonlinear absorption and their associated thresholds. We distinguish between optical damage, i.e. a reversible effect due to photo-refraction, and physical damage, i.e. an irreversible facet or bulk damage, and also show that the limitations are both intensity and average power based and hence quasi-CW modelocked operation is favored. The damage thresholds of LN vary largely in the literature (see for example [26]) and no safe operation regime can be clearly designated, particularly in the ultrashort-pulse regime. We would thus like to add information to this incomplete picture. The setups of pump source and OPO are described in section 2.A and 2.B, respectively, followed by the OPO experimental results in section 3.A and a discussion / analysis of the performance and damage issues of the MgO:PPLN crystal in section 3.B. Section 4 summarizes and concludes the findings.

\section{Experimental setup}

\section{A. Frequency-doubled, fiber-amplified, gain-switched laser diode pump source}

A schematic diagram of the MOPA pump source is shown in Fig. 1, which is very similar to the one described in detail in [27]. The seed source for the fiber amplifiers of the MOPA was a fiberpigtailed 1060nm Fabry-Perot laser diode (LD) that was gain-switched with a pulsed modulation current superimposed in a bias-tee with a DC current. A mode-selective grating and a chirped fiber Bragg grating (CFBG) were used to achieve self-seeding and to remove the inherent LD chirp, respectively. An electro-optic modulator (EOM) pulse picker was driven by a second pulse generator in order to control the pulse repetition rate over a wide range from $\sim 115 \mathrm{MHz}$ to $\sim 920 \mathrm{MHz}$. The modulation signal at $920 \mathrm{MHz}$ from the first pulse generator (for gain-switching the LD) served as an external trigger signal for the second pulse generator that reduced this repetition rate by a selectable factor of 2,4 or 8 .

The amplification was performed in four polarization-maintaining Ytterbium-doped fiber (YDF) amplifier stages, where the fiberized optical isolators (OI) and the wavelength-multiplexers were also polarization-maintaining. The two pre-amplifiers used $3 \mathrm{~m}$-long, single-mode, Yb-doped, $5 \mu \mathrm{m}$ core (NA 0.13), 130 $\mu \mathrm{m}$ cladding (NA 0.46), core-pumped fibers (PM-YDF-5/130, Nufern, East Granby, USA), pumped by $160 \mathrm{~mW}, 975 \mathrm{~nm}$ LDs in a forward and a bi-directional scheme, respectively. The third stage used the same type of fiber, but a $9 \mathrm{~m}$-long piece, which was backward clad-pumped by a $10 \mathrm{~W}, 975 \mathrm{~nm}$ LD. The final amplifier employed a $3.5 \mathrm{~m}-$ long, largemode-area, Yb-doped, $25 \mu \mathrm{m}$ core (NA 0.06), 250 $\mu \mathrm{m}$ cladding (NA 0.46), clad-pumped fiber (PLMA-YDF-25/250-VIII, Nufern, East Granby, USA), which was backward-pumped by a water-cooled, $160 \mathrm{~W}, 975 \mathrm{~nm}$ LD stack via a simple cylindrical lens combination and an aspherical lens, obtaining a launch efficiency $>80 \%$ (the only free-space section of the entire MOPA). To ensure single-mode operation, the otherwise multi-mode YDF ( $V$-number $>4$ ) was connected to the fiberized OI via a tapered splice [27] and coiled with a diameter of $\sim 7.5 \mathrm{~cm}$. 


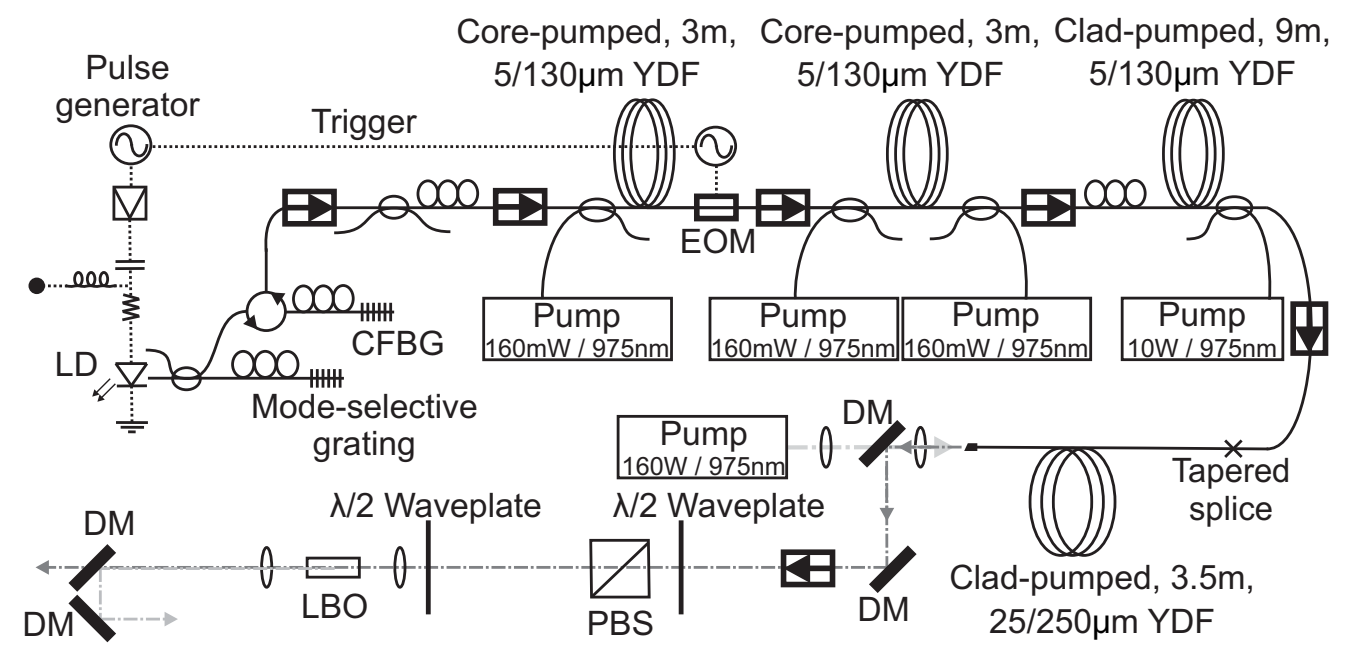

Figure 1 Schematic diagram of the fiber-amplified gain-switched laser diode MOPA system including the LBO frequency-doubling arrangement. $\mathrm{LD}=$ laser diode, $\mathrm{CFBG}=$ chirped fiber Bragg grating, EOM = electro-optic modulator, YDF = Ytterbium-doped fiber, $\mathrm{DM}=$ dichroic mirror, PBS = polarizing beamsplitter. All fibers, wavelength-division multiplexers and optical isolators were polarization-maintaining.

To prevent damage to the output facet and to avoid reflections coupling back into the fiber core, a 2mm-long, angle-polished, mode-expanding end-cap was spliced to the YDF. Dichroic mirrors (DM) were used to separate the pump and signal beams. A polarization extinction ratio of $13 \mathrm{~dB}$ at the maximum power of $\sim 78 \mathrm{~W}$ was measured at the MOPA output. The signal beam polarization was controlled with a combination of a half-waveplate and a polarizing beamsplitter (PBS), which also served as a variable amplitude attenuator. The beam quality, also measured after the optical isolator, half-waveplate and PBS, was $M^{2} \sim 1.25$ at an average output power of $35 \mathrm{~W}$. The MOPA repetition rate was set to $230 \mathrm{MHz}$, at which a maximum output power of $\sim 90 \mathrm{~W}$ before the optical isolator, half-waveplate and PBS was obtained, corresponding to $\sim 78 \mathrm{~W}$ at the MOPA output (pulse energy of $340 \mathrm{~nJ}$ ). The spectra measured at different output power levels did not exhibit significant amplified spontaneous emission (ASE) background with an optical signal-to-noise ratio of $>35 \mathrm{~dB}$. However, self-phase modulation (SPM) led to a gradual increase of the bandwidth with increasing power. At low power, the FWHM bandwidth was $0.155 \mathrm{~nm}$ and at the highest output power, the FWHM rose to $0.253 \mathrm{~nm}$ corresponding to a bandwidth-limited pulse duration of $\sim 6.5 \mathrm{ps}$ (assuming a Gaussian pulse) and therefore the measured $\sim 20 \mathrm{ps}$ pulses were approximately $3 \times$ longer than the bandwidth limit.

Frequency-doubling of the 1060nm MOPA output was performed in an AR-coated, $15 \times 3 \times 3 \mathrm{~mm}^{3}$ LBO crystal (Newlight Photonics, Toronto, Canada) cut along the $x$-axis for type I non-critical phase-matching $\left(\theta=90^{\circ}, \phi=0^{\circ}\right)$ at a temperature of $154.5^{\circ} \mathrm{C}$. At the highest fundamental input power of $77.7 \mathrm{~W}, 33 \mathrm{~W}$ of second-harmonic power was obtained corresponding to a conversion efficiency of $42 \% . M^{2}$-values of 1.65 and 1.79 in the crystal $z$ - and $y$-axis, respectively, were measured. Spectra of the second-harmonic output at different power levels and with the FWHM denoted in brackets are plotted in Fig. 2. At the highest power of $33 \mathrm{~W}$, the FWHM was $0.117 \mathrm{~nm}$, which is $\sim 6 \times$ bandwidth-limited assuming that the green pulses are also $\sim 20 \mathrm{ps}$ in duration. 
To avoid potential problems with power-dependent beam sizes and focus shifts, the MOPA and therefore the LBO output was kept at a constant power level throughout, and the amount of green power used for OPO pumping was controlled with a half-waveplate and PBS variable attenuator.

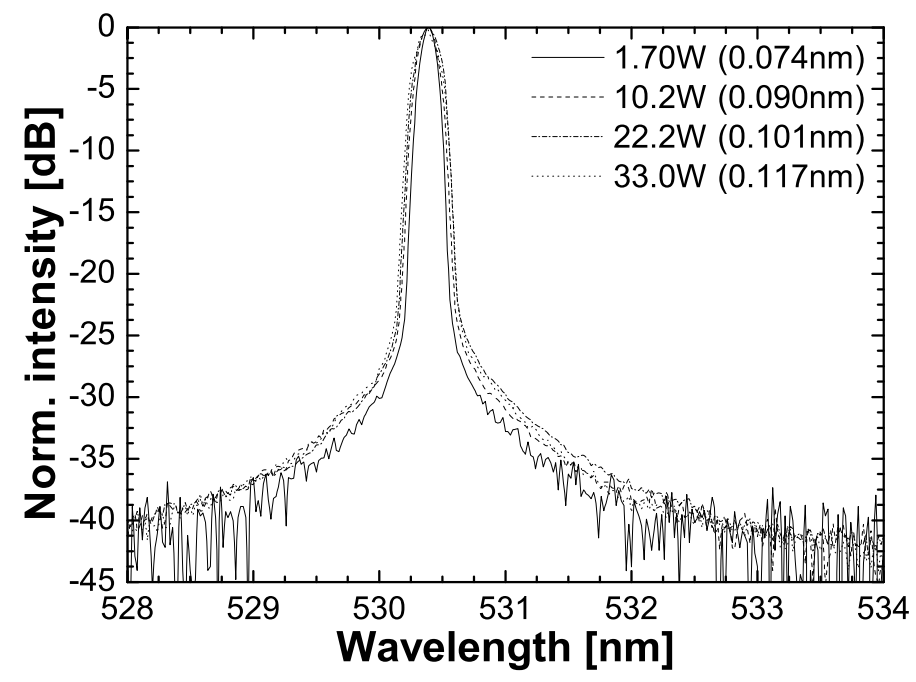

Figure 2 Normalized spectra of the second-harmonic output measured at different power levels (spectrum analyzer resolution $0.05 \mathrm{~nm}$ ). The FWHM at each power level is indicated in brackets.

\section{B. Synchronously-pumped optical parametric oscillator}

The OPO was set-up in a bow-tie ring cavity configuration as shown in Fig. 3. The congruent MgO:PPLN crystal, provided by Covesion Ltd., with a 5\% MgO-doping concentration had dimensions of $20 \times 10 \times 0.5 \mathrm{~mm}^{3}(\mathrm{~L} \times \mathrm{W} \times \mathrm{T})$ and five poled gratings with periods of $\Lambda=6.7,6.8$, $6.9,7.0,7.1 \mu \mathrm{m}$. The crystal was triple-band AR-coated on both facets for the pump, signal and idler wavelengths. The crystal was mounted in a gold-coated copper holder, which in turn was held in an oven (model PV40 from Covesion Ltd., controller TC200 from Thorlabs Inc.) with a resolution of $0.1^{\circ} \mathrm{C}$ at a temperature of $200^{\circ} \mathrm{C}$. The high temperature was a measure against photo-refraction (additional to $\mathrm{MgO}$-doping).

Two curved mirrors CM1 and CM2 with a radius of curvature of $-250 \mathrm{~mm}$, a flat mirror and a flat output coupling (OC) mirror in a bow-tie ring setup constituted the OPO resonator. The length of the ring cavity was set to $2.61 \mathrm{~m}$ corresponding to a pulse repetition rate of $115 \mathrm{MHz}$, which was half of the $230 \mathrm{MHz}$ pump pulse repetition rate, accepting that two pulses circulate at the same time in the ring cavity. This method does not pose problems in terms of OPO threshold or efficiency and has been reported before [28, 29].

CM1, CM2 and the flat mirror were highly reflective $(R>99.5 \%)$ over the signal tuning range of $650 \mathrm{~nm}$ to $1060 \mathrm{~nm}$ and highly transmissive for the pump at $530 \mathrm{~nm}(T>99 \%)$ and the idler tuning range of $1060 \mathrm{~nm}$ to $2870 \mathrm{~nm}(T>90 \%)$ resulting in singly-resonant behavior for the signal pulses. The two available OC mirrors were also highly transmissive for the pump and idler, but with transmissions of $3 \%$ and $10 \%$ over the signal wavelength range. 


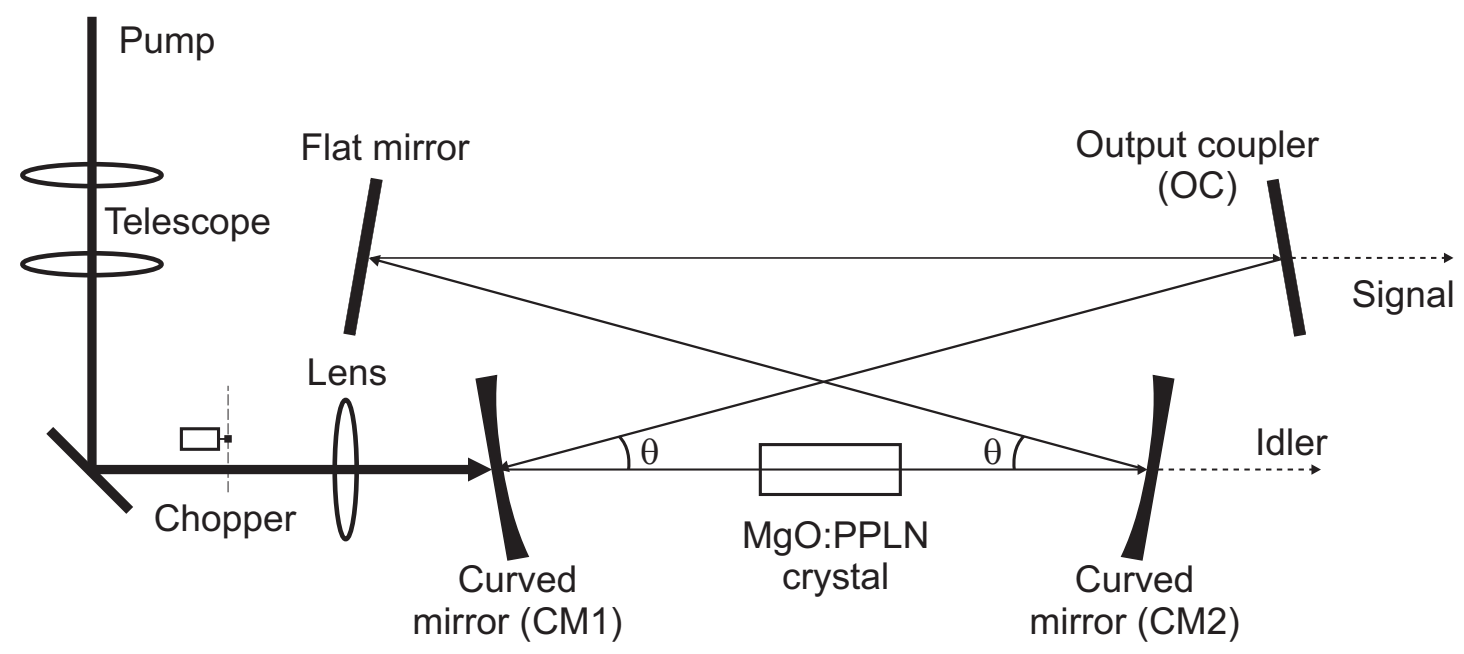

Figure 3 Layout of the singly-resonant (signal), bow-tie OPO ring resonator. The pump beam was focused with a $175 \mathrm{~mm}$ lens into the center of the MgO:PPLN after reducing its size with a telescope. The radius of curvature of CM1 and CM2 was $-250 \mathrm{~mm}$. Two output coupler mirrors with signal transmissions of $10 \%$ and $3 \%$ were available. The mechanical chopper allowed for reduction of the input average power without reduction of the pulse peak power.

The resonator was calculated to yield a signal waist radius of $\sim 42 \mu \mathrm{m}$ corresponding to a focusing parameter of $\xi_{\mathrm{s}}=0.6$ (crystal length divided by confocal parameter). A two-lens telescope was used to closely match the pump spot size to the calculated signal spot size. A pump waist with a radius of $44 \mu \mathrm{m}$ and $42 \mu \mathrm{m}$ parallel and perpendicular to the resonator plane, respectively, was obtained corresponding to a focusing parameter of $\xi_{p} \sim 0.4$.

\section{Results and discussion}

\section{A. OPO output power and pump depletion vs. pump power}

The signal and idler output power as well as the pump depletion as a function of pump power for two different OPO experiments are presented in this section. Observations of photo-refraction, GRIIRA and/or physical damage of the MgO:PPLN crystal during these experiments are discussed and analyzed in the following section 3.B, but briefly mentioned here in order to clarify the chronology of the experiments.

\section{A.1 Full average pump power as input to OPO (no mechanical chopping)}

In order to clarify the structure of this paper, we comment that 'full average power' here means that the input pump beam was not yet mechanically chopped, as was required for the experiments in section 3.A.2 ('reduced average power').

Initial oscillation of the OPO was achieved by using the $7.1 \mu \mathrm{m}$ poled grating of the MgO:PPLN and the $\mathrm{OC}$ with $T=3 \%$. In combination with the temperature of $200^{\circ} \mathrm{C}$, a signal wavelength of $808 \mathrm{~nm}$ was expected using the temperature-dependent Sellmeier equation for MgO:PPLN [30]. The average pump power at $530 \mathrm{~nm}$ was $2 \mathrm{~W}$ with an expected threshold of a few hundred milliwatts based on the same analysis as described below. The threshold, however, could not be experimentally confirmed due to the temporally unstable signal and idler output and physical damage in the center of the poled grating after only $\sim 30 \mathrm{~min}$ of operation. 
In the next step, the adjacent $7.0 \mu \mathrm{m}$ grating and considerably less average pump power of maximally $500 \mathrm{~mW}$ was used, since the oscillation threshold was expected to be relatively low. An analysis of the threshold based on [31] for low-gain (i.e. no output coupling), CW OPOs with arbitrary focusing parameter and on its extension to synchronous pumping [32] yielded an estimated average power threshold value of $\sim 100 \mathrm{~mW}$ with the assumption of $5 \%$ signal power round-trip loss (3\% output coupling, $1 \%$ crystal AR-coating reflections, $1 \%$ diffraction / scattering losses). Fig. 4 shows the signal and idler output power versus the input pump power as well as the pump depletion. The measured threshold pump power was $181 \mathrm{~mW}$ and the slope efficiencies extracted from the linear curve fits were $\sim 20 \%$ for both idler and signal. The pump depletion was not yet saturated at $500 \mathrm{~mW}$ of pump power, but reached a value between $50 \%$ and $60 \%$.

The calculated signal and idler wavelengths for $530 \mathrm{~nm}$ pump wavelength, $7.0 \mu \mathrm{m}$ crystal grating, $200^{\circ} \mathrm{C}$ crystal temperature were $825 \mathrm{~nm}$ and $1483 \mathrm{~nm}$, respectively. $M^{2}$-values of $1.41 /$ 1.34 for the idler and $1.38 / 1.44$ for the signal in the planes parallel / perpendicular to the OPO resonator were measured, respectively. The OPO output was still temporally unstable and a more careful optimization of the alignment was not feasible. After a few hours of operation, a dark spot in the center of the green pump spot at the location of the OC mirror was observed. This spot moved up and down relative to the stationary green pump spot, when the crystal was moved up and down. This was a clear indication that it was necessary to further reduce the power that the MgO:PPLN was subjected to.

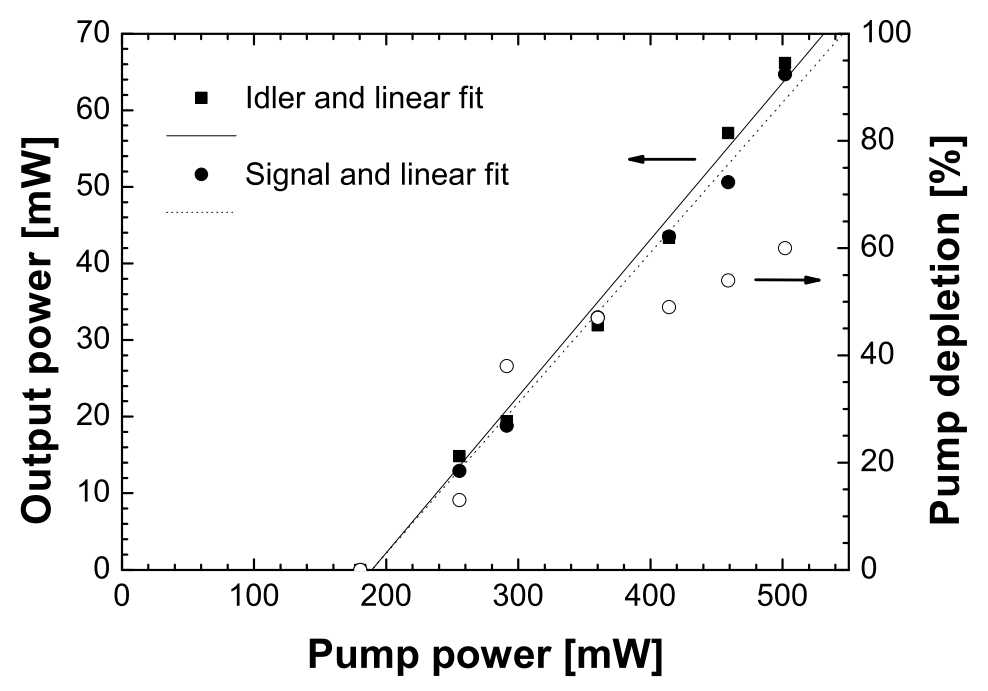

Figure 4 Signal and idler output power and pump depletion as a function of input pump power using the $7.0 \mu \mathrm{m}$ poled grating of the MgO:PPLN and a $3 \%$ OC.

\section{A.2 Reduced average pump power as input to OPO (with mechanical chopping)}

To investigate, whether the damage effects observed in the previous section were due to average power (thermally induced) or peak power, a mechanical chopper was introduced to reduce the input pump average power without reducing the pulse peak power. A mechanical chopper with a 1:9 duty cycle and a frequency of $150 \mathrm{~Hz}$ was placed in the pump beam to transmit $10 \%$ and block $90 \%$ of the total power ('transmit' period $0.67 \mathrm{~ms}$, 'block' period $6 \mathrm{~ms}$ ). Note that a reduction of the pump source repetition rate to tens of megahertz would have the same effect, but this would be less practical, since a much longer OPO cavity, for example with a fiber for signal feedback $[25,33]$, and hence a complete re-alignment of the OPO would be 
required. During the 'transmit' cycle, the peak intensity of the focused picosecond pulses remained unaltered and hence the threshold unaffected. The power values in the graphs shown in Fig. 5 and Fig. 7 are the average powers within the 'transmit' cycle, i.e. the measured powers multiplied by a factor of 10 . An MgO:PPLN grating period of $6.9 \mu \mathrm{m}$ was used yielding a signal and an idler wavelength of $845 \mathrm{~nm}$ and $1421 \mathrm{~nm}$, respectively. Due to the strongly reduced average power, no optical damage was observed in this experiment using the 3\% OC (Fig. 5), despite maintaining high peak power. The threshold was $120 \mathrm{~mW}$, the pump depletion $\sim 60 \%$ and the slope efficiencies 18\% (idler) and 14\% (signal).

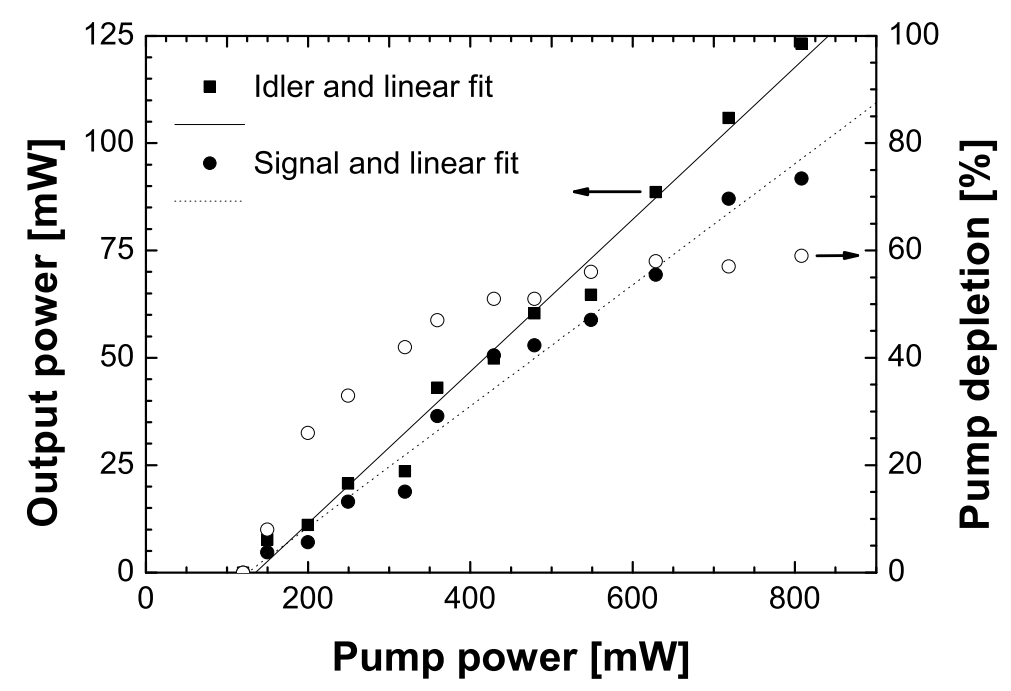

Figure 5 Output power and pump depletion versus pump power using a mechanical chopper to reduce the average input power by a factor of 10 . The $6.9 \mu \mathrm{m}$ poled grating and the $3 \%$ OC were used. All power values plotted represent the average power within the 'transmit' cycle of the chopper.

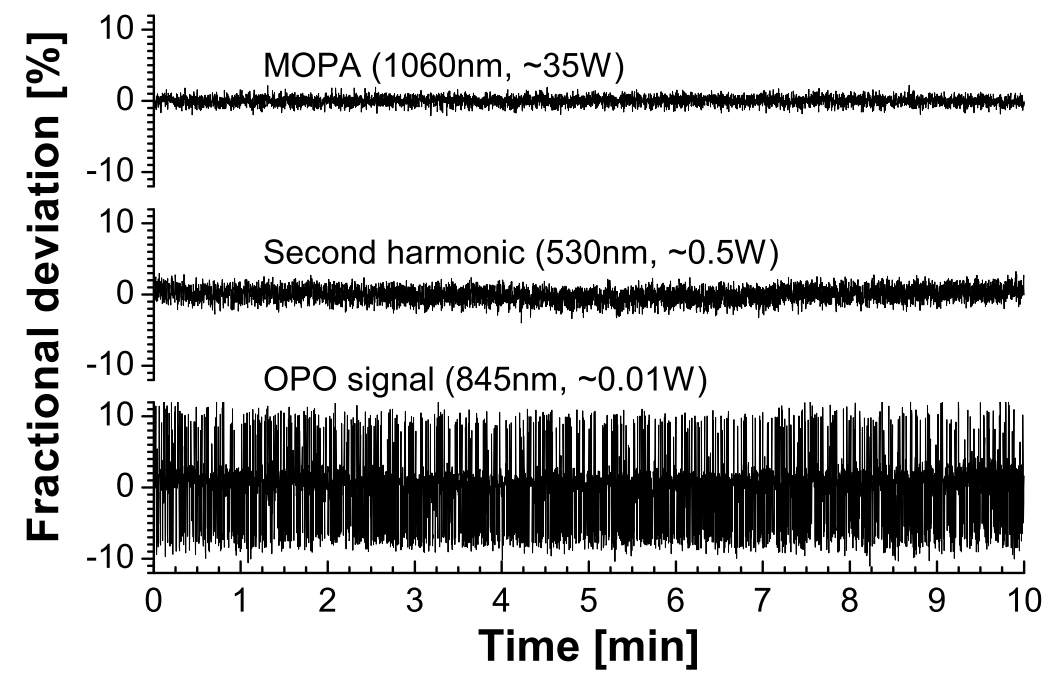

Figure 6 Fractional deviation around the mean value of the MOPA output (1060nm), the frequency-doubled output $(530 \mathrm{~nm})$ and the OPO signal output $(845 \mathrm{~nm})$ over a period of $10 \mathrm{~min}$. 


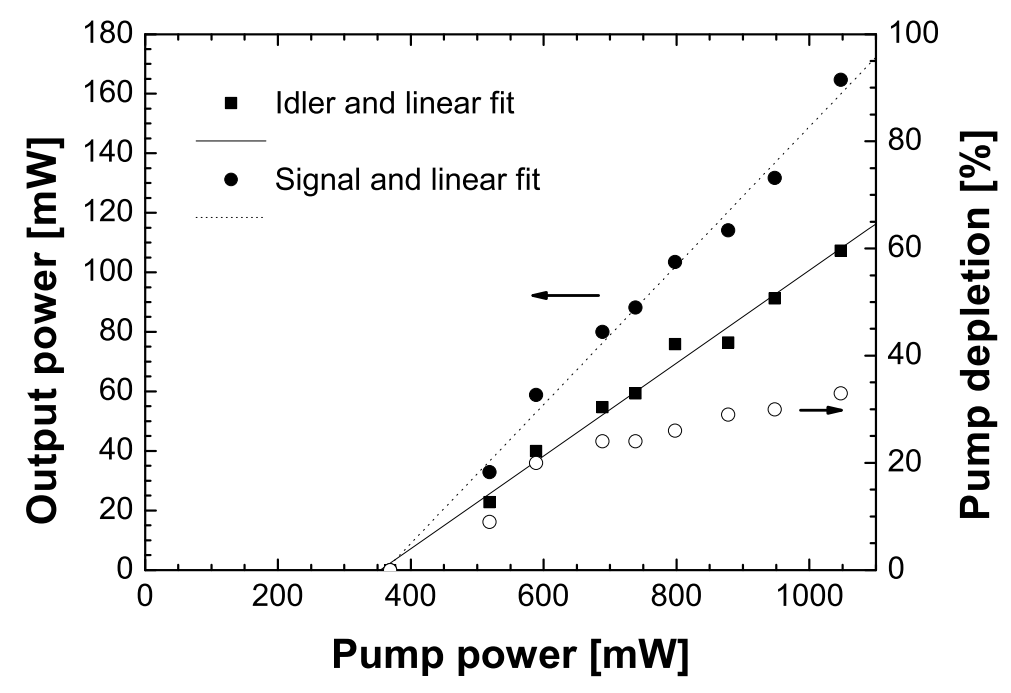

Figure 7 Same as Figure 5, but with a 10\% OC. All power values plotted represent the average power within the 'transmit' cycle of the chopper.

In order to quantify the power stability of the OPO and also the pump source, the output power from the MOPA $(1060 \mathrm{~nm})$, the frequency-doubling stage $(530 \mathrm{~nm})$ and the OPO (signal at $845 \mathrm{~nm}$ ) were recorded over a period of $10 \mathrm{~min}$ and the fractional deviations from the mean values were plotted (Fig. 6). Polarization drifts of the MOPA, air drifts and temperature fluctuations influence the transmission through the OI and PBS and the LBO conversion efficiency, which was expressed in a fractional deviation of the green power of approximately $\pm 2 \%$ compared with $\pm 1 \%$ from the MOPA. However, the fractional deviation of the OPO signal output of approximately $\pm 10 \%$ could not be solely attributed to the input pump. The OPO was enclosed in a box to reduce air drifts and temperature fluctuations to a minimum. It was suspected that photo-refraction was the main source of the OPO power instability.

The 3\% OC was then replaced by the $10 \%$ OC to extract more signal power from the resonator. The mechanical chopper was still placed in the pump beam. As shown in Fig. 7, the oscillation threshold was $369 \mathrm{~mW}$, the pump depletion saturated at $\sim 30 \%$ and the slope efficiencies were $16 \%$ (idler) and 23\% (signal). Signal and idler output powers of $165 \mathrm{~mW}$ and $107 \mathrm{~mW}$, respectively, were obtained at a pump power of $1.05 \mathrm{~W}$. The expected signal output power $P_{\mathrm{s}}$ can be calculated with [9]

$$
P_{s}=P_{\text {pump }} D \frac{\lambda_{p}}{\lambda_{s}} \frac{T_{O C}}{T_{O C}+\varepsilon_{s}},
$$

where $P_{\text {pump }}$ denotes the input pump power, $D$ the pump depletion (as a decimal number), $\lambda_{\mathrm{p}}$ and $\lambda_{\mathrm{s}}$ the pump and signal wavelengths, respectively, $T_{\mathrm{OC}}$ the $\mathrm{OC}$ transmission and $\varepsilon_{\mathrm{s}}$ the signal power round-trip loss (excluding OC transmission here). At the highest pump power of $1.05 \mathrm{~W}$, where the pump depletion was $D=0.33$, with the signal wavelength of $845 \mathrm{~nm}$ and for an estimated loss of $\varepsilon_{\mathrm{s}}=0.05$ ( $4 \%$ crystal coating reflections, $1 \%$ diffraction / scattering losses), $P_{\mathrm{s}}$ was calculated to be $145 \mathrm{~mW}$, which was in good agreement with the measured value of $165 \mathrm{~mW}$. Once again, no optical damage occurred in these experiments with the $10 \%$ OC.

Due to the output power instability of the OPO, accurate recordings of spectra with an optical spectrum analyzer were not possible. 


\section{B. Nonlinear crystal performance and damage issues}

In this section, we describe and analyze the different damage effects that occurred during the experiments of section 3.A.

We define the peak intensities $I_{\text {peak }}$ discussed in this section as

$$
I_{\text {peak }}=\frac{P_{a v}}{\Delta t \times f_{\text {rep }} \times\left(\frac{\pi}{2} w_{0}^{2}\right)},
$$

where $P_{\text {av }}$ denotes the average power, $\Delta t$ the FWHM pulse duration, $f_{\text {rep }}$ the repetition rate and $w_{0}$ the $1 / \mathrm{e}^{2}$-waist radius, assuming a Gaussian spatial beam profile. An overview of the experimental parameters and the observed damage described in the following is given in Table 1.

\begin{tabular}{|c|c|c|c|c|c|c|}
\hline \multirow{2}{*}{$\begin{array}{l}\text { Pump source } \\
\text { Average pump power } \\
\text { [W] }\end{array}$} & \multirow{2}{*}{$\begin{array}{c}\text { 532nm CW } \text { laser }^{\dagger} \\
2\end{array}$} & \multicolumn{5}{|c|}{ Frequency-doubled MOPA (20ps, 230MHz, 530nm) } \\
\hline & & 2 & 0.5 & & $1 \rightarrow 0.1 *$ & \\
\hline $\begin{array}{l}\text { 1/e } \mathrm{e}^{2} \text {-waist radius } \\
{\left[\mu^{2}\right]}\end{array}$ & $35 \times 38$ & $44 \times 42$ & $44 \times 42$ & $44 \times 42$ & $49 \times 51$ & $49 \times 51$ \\
\hline $\begin{array}{l}\text { Peak intensity } \\
{\left[\mathrm{MW} / \mathrm{cm}^{2}\right]}\end{array}$ & 0.1 & 15 & 3.75 & 7.5 & 11 & 17 \\
\hline $\begin{array}{l}\text { Crystal grating used } \\
{[\mu \mathrm{m}]}\end{array}$ & 6.7 & 7.1 & 7.0 & 6.9 & 6.8 & 6.8 \\
\hline Permanent damage? & No & $\begin{array}{c}\text { Yes } \\
\text { (optical + } \\
\text { physical) }\end{array}$ & $\begin{array}{c}\text { Yes } \\
\text { (optical) }\end{array}$ & No & $\begin{array}{c}\text { Yes } \\
\text { (optical) }\end{array}$ & $\begin{array}{c}\text { Yes } \\
\text { (optical + } \\
\text { physical) }\end{array}$ \\
\hline
\end{tabular}

'no OPO operation

*mechanically chopped at $1: 9$ duty cycle to $0.1 \mathrm{~W}$

Table 1 Summary of the different OPO pump regimes and damage effects observed (optical $=$ dark spots $/$ beam distortion during operation, physical $=$ degradation of the crystal visible with a microscope).

\section{B.1 Crystal performance under the influence of the full average pump power}

This section refers to the observations made during the experiments of section 3.A.1, where no mechanical chopper for the pump beam was used.

With $2 \mathrm{~W}$ of average pump power, which corresponds to a peak intensity of $\sim 15 \mathrm{MW} / \mathrm{cm}^{2}$, incident for approximately $30 \mathrm{~min}$ to the $7.1 \mu \mathrm{m}$ grating of the MgO:PPLN crystal, permanent physical damage was visible with the unaided eye. The microscope image in Fig. 8 clearly shows individual, closely spaced damage tracks within a cigar-shaped volume in the center of the MgO:PPLN crystal. This has been reported before and photo-refraction or self-focusing was suspected to cause the damage [34]. The damage is clearly associated with the high intensity pumping region, with the pump waist being located at the center of the crystal. With the equation for the critical power of self-focusing given in [35], a critical power of $\sim 22 \mathrm{~kW}$ is calculated using the nonlinear refractive index $8.3 \times 10^{-19} \mathrm{~m}^{2} / \mathrm{W}$ at $532 \mathrm{~nm}$ [26], the linear refractive index 2.3 at $530 \mathrm{~nm}$ and assuming a Gaussian beam profile. The peak power of the 20ps long pump pulses was only $435 \mathrm{~W}$ and, thus, self-focusing can be safely ruled out as the cause for physical damage. 


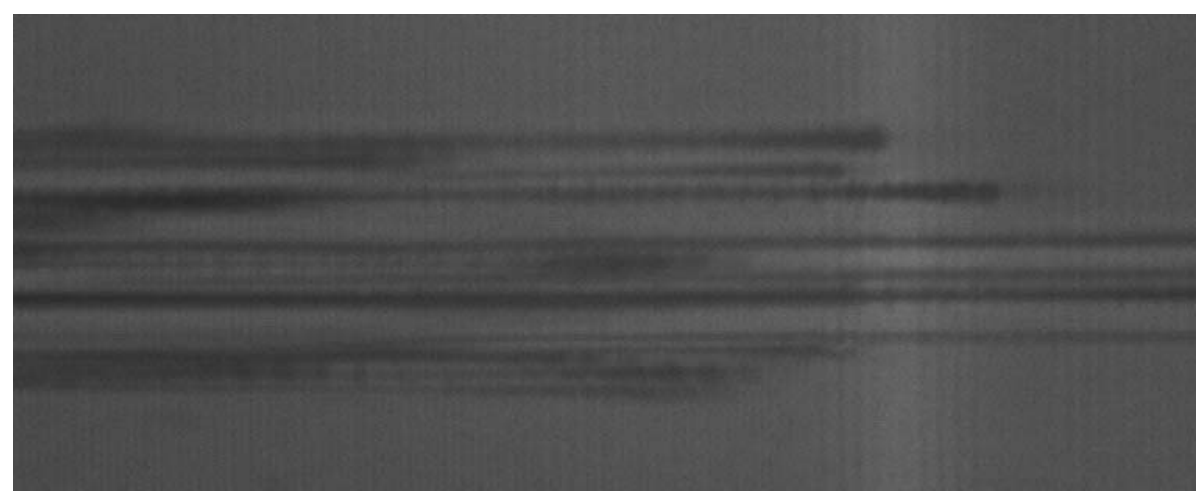

Figure 8 Microscope image of the damage within the $7.1 \mu \mathrm{m}$ poled grating, where individual damage tracks are clearly visible.

Additionally, the transmitted green pump beam was distorted and had a dark center (region of physical damage) as observed in the green spot on the OC mirror. This beam distortion was previously described as a consequence of photo-refraction $[10,23]$. The dark spot within the green spot could be a preceding, more sensitive sign of the physical track formation before it can be seen with a microscope and was possibly not only due to photo-refraction, but also due to GRIIRA. Since a signal and an idler wave in the infrared were generated, GRIIRA could have led to a thermal loading of the crystal and the associated physical damage. The inherent infrared absorption of LN can increase by up to a factor of five with green light present [14] and is quadratically dependent on the green power, but can recover to its initial value after $\sim 20$ s [13] before physical damage occurs. According to findings in [14], a higher MgO-doping concentration of $>6 \%$ could help to avoid GRIIRA, because a threshold-like behavior in terms of optical damage has been reported. For a higher concentration, all of the intrinsic $\mathrm{Nb}^{5+}$ anti-site defects $\left(\mathrm{Nb}^{5+}\right.$-ions at the lattice location of $\mathrm{Li}^{+}$-ions) of congruent $\mathrm{LN}$ are replaced by $\mathrm{Mg}^{2+}$-ions. In general, $\mathrm{Nb}^{5+}$ anti-site defects act as electron traps and locally change the absorption properties, referred to as polaron absorption, which is inhibited when $\mathrm{Mg}^{2+}$-ions occupy these anti-site defects. However, MgO:PPLN crystals with doping concentrations $>5 \%$ suffer from growth defects and can not be grown at high quality, which also impairs the subsequent poling process. Hence, an optimum concentration of $5 \%$ is typically used by commercial suppliers. Alternatively, a stoichiometric crystal composition with balanced $\mathrm{Nb}$ and Li concentrations could decrease GRIIRA due to the absence of anti-site defects. To confirm whether or not GRIIRA caused the physical damage, an unpoled region of the MgO:PPLN sample was pumped for $30 \mathrm{~min}$ under the same conditions as above, i.e. $2 \mathrm{~W}$ of average power and $\sim 15 \mathrm{MW} / \mathrm{cm}^{2}$ of peak intensity. Since no parametric oscillation can occur in an unpoled region, no signal or idler in the IR can be generated and hence green-induced IR absorption can not occur. Physical damage of the unpoled region was observed again, which is evidence that processes other than only GRIIRA contribute to the physical damage. Similar to $\mathrm{Nb}^{5+}$ anti-site defects, lattice impurities such as $\mathrm{Fe}^{2+}$-ions can facilitate both linear and nonlinear (e. g. two-photon) absorption [14, 15], which can potentially be a damage contributor [36]. Although the MgO:PPLN sample was of high quality, impurities on the order of parts-per-million naturally occur [12, 36, 37].

The $7.0 \mu \mathrm{m}$ grating that was pumped with a $4 \times$ lower average power of $0.5 \mathrm{~W}$ (after damage of the $7.1 \mu \mathrm{m}$ grating at $2 \mathrm{~W}$ ) did not show any signs of visible physical damage after a few hours of OPO operation. However, the beam distortion and the dark spot within the green spot on the OC as described above were again existent. The lower average power and the lower peak intensity of $3.75 \mathrm{MW} / \mathrm{cm}^{2}$ did not trigger GRIIRA at a level that could have caused the 
catastrophic thermal loading and physical damage seen before (quadratic scaling law). Photorefraction appears to be the dominating effect here. A comparison with reported threshold intensities for photo-refraction of $2 \mathrm{MW} / \mathrm{cm}^{2}$ for a congruent $5 \% \mathrm{MgO}: \mathrm{PPLN}$ sample [16] and $>8 \mathrm{MW} / \mathrm{cm}^{2}$ for a congruent $6 \% \mathrm{MgO}: \mathrm{PPLN}$ sample [14] $(\mathrm{CW}$, green light, focused beams, no OPO operation) shows that the intensity of this experiment was on the same order of magnitude. On the other hand, damage thresholds vary largely in the literature based on the pump source parameters. Also, definitions of damage vary considerably in the published literature with some papers referring to damage as the onset of measurable changes in the refractive index, whilst others refer to damage as irreversible physical damage. Depending on the level of exposure, the effects of photo-refraction can be reversed by annealing the crystal for a few hours. However, the dark spot was still existent after $2 \mathrm{~h}$ of annealing at $220^{\circ} \mathrm{C}$ and a recovery period of many days (without using the crystal in the OPO). Thus in this case, the damage appears to be irreversible suggesting not only optical, but also physical damage.

\section{B.2 Crystal performance under the influence of reduced average pump power}

This section refers to the observations made during the experiments of section 3.A.2, where a mechanical chopper in the pump beam reduced the average pump power.

The experiments with a mechanically chopped pump beam were carried out in the next poled grating of the MgO:PPLN with a period of $6.9 \mu \mathrm{m}$. The importance of this experiment was to isolate the effect of high peak power from the effect of high average power on the damage and to investigate their impacts. A maximum of $\sim 1 \mathrm{~W}$ of average pump power before the chopper was $10 \times$ reduced to $\sim 0.1 \mathrm{~W}(1: 9$ duty cycle) and then focused into the crystal. The peak intensity before and after the chopper was $7.5 \mathrm{MW} / \mathrm{cm}^{2}$, since the pulses within the $0.67 \mathrm{~ms}-$ long, $10 \%$ 'transmit' cycle were not affected by the chopping. Under these circumstances, i.e. with a true input average pump power of $0.1 \mathrm{~W}$, the OPO was operated without any permanent damage or dark spots observed. The 6ms-long chopper 'block' cycle was too short for a recovery of the GRIIRA effect, which has a time constant of $\sim 20$ s [13], but the significantly reduced average power avoided the physical damage. The strong signal and idler output power fluctuations (Fig. 6) indicated that some low-level photo-refraction was still present. Again, the 6ms 'block' cycle was not long enough to allow for a natural recovery of the photo-refractive effect, i.e. a complete diffusion of the free charge carriers, since the time constants for this process, even at elevated temperatures, are orders of magnitude larger. With the intensity of $7.5 \mathrm{MW} / \mathrm{cm}^{2}$ being $2 \times$ higher than in the previous experiment, the damage process appears to require high average power and not just high intensity.

\section{Optimum operational regime}

For a more exact determination of the damage threshold, we carried out a systematic investigation starting from the situation as given in column 5 of Table 1 with $0.1 \mathrm{~W}$ of average power as input to the crystal, where no damage was observed. The average power was kept constant and the peak intensity was successively increased. This independent control was achieved by using a half-waveplate and polarizing beamsplitter to increase the average power and hence the peak intensity followed by a mechanical chopper with wheels of decreasing duty cycle to only reduce the average power but not the peak intensity of the pulses within the 'transmit' cycle. After $1 \mathrm{~h}$ of operation (signal and idler present) using the previously unused $6.8 \mu \mathrm{m}$ grating and a pump pulse intensity of $\sim 11 \mathrm{MW} / \mathrm{cm}^{2}$, the beam distortion and dark spot effect were observed, but no physical damage was generated. At an even higher intensity of $\sim 17 \mathrm{MW} / \mathrm{cm}^{2}$, physical damage occurred after only a few minutes in that same grating. Therefore, the peak intensity damage threshold can be pin-pointed to a range of around $7.5 \mathrm{MW} / \mathrm{cm}^{2}$ to $11 \mathrm{MW} / \mathrm{cm}^{2}$. 
In order to further confirm the assumption that both average power and peak intensity lead to crystal damage, the influence of pulse peak power / intensity on the described damage was completely eliminated by using a frequency-doubled $(532 \mathrm{~nm})$, diode-pumped, solid-state $\mathrm{CW}$ laser with up to $2 \mathrm{~W}$ of average power focused to a spot with $1 / \mathrm{e}^{2}$-radius of $35 \mu \mathrm{m} \times 38 \mu \mathrm{m}$ inside the MgO:PPLN (intensity at the waist $\sim 0.1 \mathrm{MW} / \mathrm{cm}^{2}$ ). If it was exclusively the average power of $2 \mathrm{~W}$ causing the physical damage, then using a $2 \mathrm{~W}$ green $\mathrm{CW}$ laser should also lead to this type of damage. Without operating the nonlinear crystal in an OPO but with simple transmission of the focused green beam through the $6.7 \mu \mathrm{m}$ grating for a period of $1 \mathrm{~h}$, this grating did not exhibit the previously observed physical damage (closely spaced tracks) or dark spots. Therefore, the damage threshold regarding average power only (no pulses) exceeds $2 \mathrm{~W}$. This observation is supported by OPO and SHG publications in the CW regime reporting several Watts of green light [17-20].

Table 1 gives a summary of the different OPO pump regimes used in the experiments and the parameters involved. In this context, optical damage means the dark spots within and the beam distortion of the transmitted green pump beam (typically reversible), whereas physical damage means a physical degradation of the crystal structure visible under a microscope (irreversible).

After all the experiments were carried out, the crystal coatings were examined with a microscope and no anomalies were seen. Thus, all the effects described in this section were not caused by problems with the coatings.

\section{Discussion}

The damage observed can be classified into two categories: optical damage (dark spots and beam distortion) and physical damage (degradation of the crystal structure) depending on the average power and peak intensity conditions. However, it can not be ruled out that the optical damage is only a preceding, more sensitive sign of the physical damage before it can be seen with a microscope. Furthermore, instabilities due to photo-refraction were always present and could be quantified with an output power fluctuation of $\pm 10 \%$ of the OPO signal beam.

It was found that high pulse peak intensities were required to damage the crystal, since an experiment with a green CW pump laser did not cause damage up to an average power of $2 \mathrm{~W}$ (the intensity at the focus was $0.1 \mathrm{MW} / \mathrm{cm}^{2}$ ). However, a reduction of the average power seemed to be beneficial in that for an average power of $0.1 \mathrm{~W}$, peak intensities of $11 \mathrm{MW} / \mathrm{cm}^{2}$ were required to observe damage, whereas only $3.75 \mathrm{MW} / \mathrm{cm}^{2}$ led to damage, when the average power was $0.5 \mathrm{~W}$.

It was also found that the green pump light alone led to optical and physical damage, which was demonstrated by subjecting an unpoled region of the MgO:PPLN sample to $2 \mathrm{~W}$ and $15 \mathrm{MW} / \mathrm{cm}^{2}$ of pump light for around $30 \mathrm{~min}$, after which the crystal was damaged. It was argued that if no parametric oscillation is possible and hence no signal and idler in the infrared is present, no green-induced IR absorption should be possible. From this observation, we conclude that while GRIIRA may have contributed to the damage during OPO operation, it was not the sole damage process.

Given that the damage process appears to only require intense green light and not the presence of infrared light, we speculate that the process is initially due to nonlinear absorption. The dependence of the damage process on average power as well as intensity suggests that linear absorption, perhaps as a result of defects formed by the high intensity visible light, also plays a role. It has previously been shown that lattice impurities, primarily $\mathrm{Fe}$-ions on the parts-permillion scale, can facilitate linear and nonlinear absorption [12, 14, 15, 36, 37]. 


\section{Conclusions}

In summary, we have demonstrated a synchronously pumped OPO based on MgO:PPLN producing $\sim 165 \mathrm{~mW}$ (signal, $845 \mathrm{~nm}$ ) and $\sim 107 \mathrm{~mW}$ (idler, $1421 \mathrm{~nm}$ ) of average power for $\sim 1 \mathrm{~W}$ of green pump power at $530 \mathrm{~nm}$. The oscillation threshold was $369 \mathrm{~mW}$ and the pump depletion saturated at $\sim 30 \%$. The pump source consisted of a $20 \mathrm{ps}$, frequency-doubled, fiber-amplified, gain-switched laser diode MOPA system operating at a repetition rate of $230 \mathrm{MHz}$. This OPO can theoretically generate signal and idler wavelengths between $800 \mathrm{~nm}$ and $900 \mathrm{~nm}$ and $1.28 \mu \mathrm{m}$ and $1.54 \mu \mathrm{m}$, respectively, and thus is an attractive alternative to Ti:Sapphire laser, currently the preferred choice for tunable ultrashort pulses in the near-infrared. However, operation, especially at higher pump power, was complicated and even prevented due to well-known effects occurring in LN such as photo-refraction and GRIIRA. It was found that a combination of both high average power and high peak intensities led to crystal damage.

An investigation of crystal damage mechanisms, when MgO:PPLN is subjected to light pulses in the visible (as opposed to CW operation with several Watts of power, where no such effects are observed), was carried out with the result that the observed damage required peak intensities of around $10 \mathrm{MW} / \mathrm{cm}^{2}$. But at the same time, an influence of the average power to the damage was evident and a reduction of the average power appeared to be beneficial for the same peak intensity. Photo-refraction, GRIIRA and nonlinear absorption were identified as possible damage processes with nonlinear absorption being the most likely process for physical damage of the crystal structure.

We confirmed the already wide-spread insight that LN used in ultrashort-pulse nonlinear devices with visible light suffers from detrimental effects, which limit the device efficiency and beam quality, but we were able to add important information as to where the limits and damage thresholds lie. This information can be added to the 'map' of safe operation regimes in terms of pulse duration, repetition rates, power levels, peak intensities and wavelengths and we hope that it might prove useful for future experiments. Nevertheless, LN is still an interesting crystal compared to bulk crystals such as LBO for use in green-pumped, ultrashort-pulse OPOs due to its advantageous properties such as the high nonlinear coefficient $d_{\text {eff }}=17 \mathrm{pm} / \mathrm{V}$, the possibility of engineered phase-matching by periodic poling, the well-known physical and optical properties, the wide availability and the low price. A viable option for an efficient, pulsed, greenpumped OPO based on MgO:PPLN could be a low pump repetition rate of a few megahertz and a long but still compact fiber-feedback OPO resonator [25, 33], where the average power is low but the peak intensity is relatively high.

\section{Acknowledgements}

This work was supported by the Engineering and Physical Sciences Research Council (EPSRC) grant EP/F032218/1. F. Kienle acknowledges the support of an EPSRC studentship.

\section{References}

1. C. Cleff, J. Epping, P. Gross, and C. Fallnich, "Femtosecond OPO based on LBO pumped by a frequency-doubled Yb-fiber laser-amplifier system for CARS spectroscopy," Applied Physics B: Lasers and Optics 103, 795-800 (2011).

2. C. L. Evans, E. O. Potma, M. Puoris'haag, D. Côté, C. P. Lin, and X. S. Xie, "Chemical imaging of tissue in vivo with video-rate coherent anti-Stokes Raman scattering microscopy," Proceedings of the National Academy of Sciences of the United States of America 102, 16807-16812 (2005). 
3. F. Ganikhanov, S. Carrasco, X. Sunney Xie, M. Katz, W. Seitz, and D. Kopf, "Broadly tunable dual-wavelength light source for coherent anti-Stokes Raman scattering microscopy," Opt. Lett. 31, 1292-1294 (2006).

4. K. Kieu, B. G. Saar, G. R. Holtom, X. S. Xie, and F. W. Wise, "High-power picosecond fiber source for coherent Raman microscopy," Opt. Lett. 34, 2051-2053 (2009).

5. S. W. Hell and J. Wichmann, "Breaking the diffraction resolution limit by stimulated emission: stimulated-emission-depletion fluorescence microscopy," Opt. Lett. 19, 780782 (1994).

6. S. D. Butterworth, S. Girard, and D. C. Hanna, "High-power, broadly tunable all-solidstate synchronously pumped lithium triborate optical parametric oscillator," J. Opt. Soc. Am. B 12, 2158-2167 (1995).

7. M. Jurna, J. P. Korterik, H. L. Offerhaus, and C. Otto, "Noncritical phase-matched lithium triborate optical parametric oscillator for high resolution coherent anti-Stokes Raman scattering spectroscopy and microscopy," Applied Physics Letters 89, 251116251113 (2006).

8. J. D. Kafka, M. L. Watts, and J. W. Pieterse, "Synchronously pumped optical parametric oscillators with $\mathrm{LiB}_{3} \mathrm{~B}_{5}$, " J. Opt. Soc. Am. B 12, 2147-2157 (1995).

9. T. W. Tukker, C. Otto, and J. Greve, "Design, optimization, and characterization of a narrow-bandwidth optical parametric oscillator," J. Opt. Soc. Am. B 16, 90-95 (1999).

10. A. Ashkin, G. D. Boyd, J. M. Dziedzic, R. G. Smith, A. A. Ballman, J. J. Levinstein, and K. Nassau, "Optically-induced refractive index inhomogeneities in $\mathrm{LiNbO}_{3}$ and $\mathrm{LiTaO}_{3}$," Applied Physics Letters 9, $72-74$ (1966).

11. R. W. Boyd, Nonlinear Optics, 2nd ed. (Academic Press, San Diego, 2002), p. 576.

12. F. Jermann, M. Simon, and E. Krätzig, "Photorefractive properties of congruent and stoichiometric lithium niobate at high light intensities," J. Opt. Soc. Am. B 12, 2066-2070 (1995).

13. R. G. Batchko, D. R. Weise, T. Plettner, G. D. Miller, M. M. Fejer, and R. L. Byer, "Continuous-wave 532-nm-pumped singly resonant optical parametric oscillator based on periodically poled lithium niobate," Opt. Lett. 23, 168-170 (1998).

14. Y. Furukawa, K. Kitamura, A. Alexandrovski, R. K. Route, M. M. Fejer, and G. Foulon, "Green-induced infrared absorption in $\mathrm{MgO}$ doped $\mathrm{LiNbO}_{3}$," Applied Physics Letters 78, 1970-1972 (2001).

15. J. Hirohashi, V. Pasiskevicius, S. Wang, and F. Laurell, "Picosecond blue-light-induced infrared absorption in single-domain and periodically poled ferroelectrics," Journal of Applied Physics 101, 033105 (2007).

16. M. Nakamura, S. Higuchi, S. Takekawa, K. Terabe, Y. Furukawa, and K. Kitamura, "Optical damage resistance and refractive indices in near-stoichiometric $\mathrm{MgO}$-doped $\mathrm{LiNbO}_{3}$," Jpn. J. Appl. Phys. Part 2 - Lett. 41, L49-L51 (2002).

17. D.-H. Lee, S. K. Kim, S.-N. Park, H. Su Park, J. Y. Lee, and S.-K. Choi, "Continuouswave 532nm pumped MgO:PPLN optical parametric oscillator with external power regulation and spatial mode filtering," Appl. Opt. 48, 37-42 (2009).

18. S. Zaske, D. H. Lee, and C. Becher, "Green-pumped cw singly resonant optical parametric oscillator based on MgO:PPLN with frequency stabilization to an atomic resonance," Applied Physics B: Lasers and Optics 98, 729-735 (2010).

19. H. Furuya, A. Morikawa, K. Mizuuchi, and K. Yamamoto, "High-beam-quality continuous wave $3 \mathrm{~W}$ green-light generation in bulk periodically poled $\mathrm{MgO}: \mathrm{LiNbO}_{3}$," Japanese Journal of Applied Physics Part 1-Regular Papers Brief Communications \& Review Papers 45, 6704-6707 (2006).

20. C. B. E. Gawith, Covesion Ltd., Romsey, SO51 9DG, UK, personal communication (2011). 
21. V. Pruneri, S. D. Butterworth, and D. C. Hanna, "Low-threshold picosecond optical parametric oscillation in quasi-phase-matched lithium niobate," Applied Physics Letters 69, 1029-1031 (1996).

22. P. F. Chimento, M. Jurna, H. S. P. Bouwmans, E. T. Garbacik, L. Hartsuiker, C. Otto, J. L. Herek, and H. L. Offerhaus, "High-resolution narrowband CARS spectroscopy in the spectral fingerprint region," J. Raman Spectrosc. 40, 1229-1233 (2009).

23. L. E. Myers, R. C. Eckardt, M. M. Fejer, R. L. Byer, W. R. Bosenberg, and J. W. Pierce, "Quasi-phase-matched optical parametric oscillators in bulk periodically poled $\mathrm{LiNbO}_{3}$," J. Opt. Soc. Am. B 12, 2102-2116 (1995).

24. F. Kienle, K. K. Chen, S.-U. Alam, C. B. E. Gawith, J. I. Mackenzie, D. C. Hanna, D. J. Richardson, and D. P. Shepherd, "High-power, variable repetition rate, picosecond optical parametric oscillator pumped by an amplified gain-switched diode," Opt. Express 18, 7602-7610 (2010).

25. F. Kienle, P. Siong Teh, S.-U. Alam, C. B. E. Gawith, D. C. Hanna, D. J. Richardson, and D. P. Shepherd, "Compact, high-pulse-energy, picosecond optical parametric oscillator," Opt. Lett. 35, 3580-3582 (2010).

26. D. N. Nikogosyan, Nonlinear optical crystals: a complete survey, 1st ed. (Springer, Berlin / Heidelberg, 2005), p. 427.

27. K. K. Chen, J. H. V. Price, S.-U. Alam, J. R. Hayes, D. J. Lin, A. Malinowski, and D. J. Richardson, "Polarisation maintaining 100W Yb-fiber MOPA producing $\mu \mathrm{J}$ pulses tunable in duration from 1 to 21 ps," Opt. Express 18, 14385-14394 (2010).

28. S. Lecomte, R. Paschotta, M. Golling, D. Ebling, and U. Keller, "Synchronously pumped optical parametric oscillators in the $1.5-\mu \mathrm{m}$ spectral region with a repetition rate of 10 GHz," J. Opt. Soc. Am. B 21, 844-850 (2004).

29. X. P. Zhang, J. Hebling, A. Bartels, D. Nau, J. Kuhl, W. W. Rühle, and H. Giessen, "1GHz-repetition-rate femtosecond optical parametric oscillator," Applied Physics Letters 80, 1873-1875 (2002).

30. O. Gayer, Z. Sacks, E. Galun, and A. Arie, "Temperature and wavelength dependent refractive index equations for $\mathrm{MgO}$-doped congruent and stoichiometric $\mathrm{LiNbO}_{3}$," Applied Physics B: Lasers and Optics 91, 343-348 (2008).

31. S. Guha, "Focusing dependence of the efficiency of a singly resonant optical parametric oscillator," Applied Physics B: Lasers and Optics 66, 663-675 (1998).

32. M. J. McCarthy and D. C. Hanna, "All-solid-state synchronously pumped optical parametric oscillator," J. Opt. Soc. Am. B 10, 2180 (1993).

33. T. Südmeyer, E. Innerhofer, F. Brunner, R. Paschotta, T. Usami, H. Ito, S. Kurimura, K. Kitamura, D. C. Hanna, and U. Keller, "High-power femtosecond fiber-feedback optical parametric oscillator based on periodically poled stoichiometric $\mathrm{LiTaO}_{3}$," Opt. Lett. 29, 1111-1113 (2004).

34. D. H. Titterton, J. A. C. Terry, D. H. Thorne, I. R. Jones, and D. Legge, "Observation of damage in PPLN," in Growth, Fabrication, Devices, and Applications of Laser and Nonlinear Materials, 2001), 5-13.

35. G. Fibich and A. L. Gaeta, "Critical power for self-focusing in bulk media and in hollow waveguides," Opt. Lett. 25, 335-337 (2000).

36. J. R. Schwesyg, A. Markosyan, M. C. C. Kajiyama, M. Falk, D. H. Jundt, K. Buse, and M. M. Fejer, "Optical loss mechanisms in magnesium-doped lithium niobate crystals in the 300 to $2950 \mathrm{~nm}$ wavelength range," in Advanced Solid-State Photonics (ASSP), 2011),

37. D. A. Bryan, R. Gerson, and H. E. Tomaschke, "Increased optical damage resistance in lithium niobate," Applied Physics Letters 44, 847-849 (1984). 